\title{
Rapid cometary sample return enabled by low-cost, 10- gram interplanetary spacecraft
}

Kristofer S.J. Pister ${ }^{1}$, Andrew J. Westphal ${ }^{2}$

${ }^{1}$ Berkeley Sensor and Actuator Center, ${ }^{2}$ Space Sciences Laboratory

U.C. Berkeley 


\section{Rapid cometary sample return enabled by low-cost, 10- gram interplanetary spacecraft}

Kristofer S.J. Pister ${ }^{1}$, Andrew J. Westphal ${ }^{2}$

${ }^{1}$ Berkeley Sensor and Actuator Center, ${ }^{2}$ Space Sciences Laboratory

U.C. Berkeley

\section{Executive Summary}

Existing cell phone technology and recent advances in microelectromechanical systems (MEMS) enable unprecedented capabilities in space. Fleets of thousands of tiny interplanetary spacecraft, navigating using solar sails, will soon enable rapid exploration of the inner Solar System. Return of pristine cometary material is a high NASA priority, as reflected in the Phase A selections from the most recent New Frontiers competition. Here, we describe a mission architecture using miniature interplanetary spacecraft that could rapidly return pristine cometary material from dozens of Jupiter Family Comets (JFCs) at a fraction of the cost of a sample return mission from a single JFC using a more traditional approach. Each spacecraft would weigh approximately ten grams, and would navigate using a solar sail. All of the components of such miniaturized spacecraft have been demonstrated in the laboratory or in commercial applications, but will require investment to qualify for space applications.

\section{Importance of pristine cometary sample return}

Comets contain the building blocks of the solar system, preserved in deep freeze for 4.568 billion years. Study of cometary samples returned by the Stardust mission and collected in the stratosphere has demonstrated that the earliest history of the Solar System is recorded in these tiny samples. In 2006, the NASA Stardust spacecraft returned the first cometary samples to terrestrial laboratories, resulting in $>140$ publications and the rewriting our understanding of the earliest history of the solar system. However, neither of the existing collections of cometary materials are pristine, but were modified by the collection method capture at $6.1 \mathrm{~km} / \mathrm{sec}$ in aerogel and $\mathrm{Al}$ foil collectors in the case of Stardust, and atmospheric entry at $\sim 10 \mathrm{~km} / \mathrm{sec}$ in the case of the stratospheric collection. In particular, the organics and the finest-grained materials, either did not survive or were severely altered. As a result, critically important pieces of the puzzle are missing. Cometary organics are thermally fragile and as a result are poorly understood. The delivery of organics to the prebiotic Earth may have played a key role in making it habitable and in the origin of life [1, 2, 3]. Increasingly sophisticated laboratory techniques, largely enabled by high-resolution synchrotron infrared and soft x-ray microprobes, will enable detailed analysis of organics within their petrographic contexts. BLISS spacecraft could be used to return unaltered organics and fine-grained materials in coma dust samples from dozens of comets, by collecting the samples at $\mathrm{m} / \mathrm{sec}$ capture speeds, rather than $\mathrm{km} / \mathrm{sec}$ speeds.

Sample return of pristine cometary material was identified as a high priority in the most recent Planetary Decadal Survey, and a comet sample return mission — from a single comet - 
was one of two finalists in the most recent New Frontiers (\$1B-class) competition. Even with an aggressive schedule, cometary samples would be expected no earlier than the mid 2040's with a New Frontiers approach. In contrast, a fleet of 10 gram interplanetary spacecraft has the potential to collect cometary samples from dozens of Jupiter-family comets within the next decade, at a small fraction of the cost of a NF mission to a single comet.

Because cometary materials are complex on the submicron scale[4], large samples are not required for cometary sample return: the thousands of $\sim 10 \mu \mathrm{m}$ rocks and abundant organic materials would keep the cosmochemistry community busy for decades. Such a sample could be collected in less than a day from a typical cometary coma, using a $1 \mathrm{~cm}^{2}$ collector with a MEMS-actuated door.

\section{BLISS: Berkeley Low-cost Interplanetary Solar Sails}

Existing cell phone technology and recent advances in microelectromechanical systems (MEMS) enable unprecedented capabilities in space. Fleets of thousands of tiny interplanetary spacecraft, navigating using solar sails, will soon enable rapid exploration of the inner Solar System. Many applications are possible, including optical reconnaissance of thousands of Near-Earth Objects; establishment of a peer-to-peer communication network to pass data around the inner solar system; and synoptic observations of the Earth's magnetosphere, or that of other planetary bodies, at thousands to millions of locations. Here, we focus on miniaturized spacecraft optimized for rapid cometary sample return.
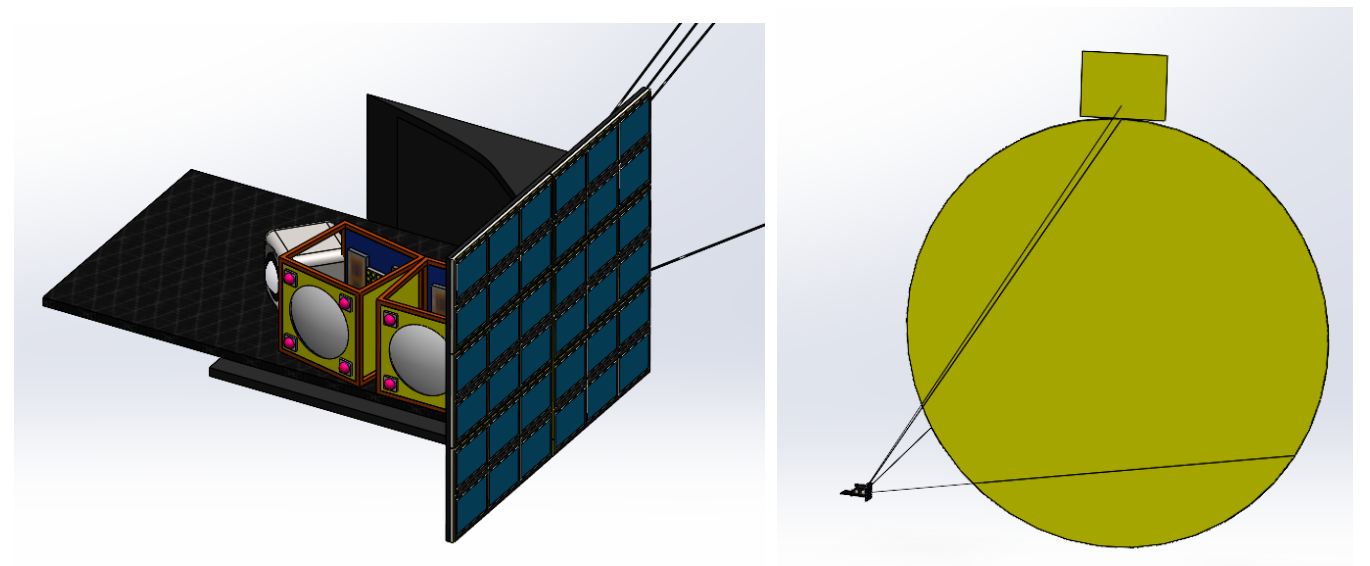

Figure 1: BLISS spacecraft for rapid sample return of pristine cometary material. Left: Spacecraft body with solar cells, radiator fin, optical comms, gumstix computer. Right: body connected to sail via shroud control lines.

The spacecraft would consist of a miniature spacecraft bus, connected by shrouds to a solar sail (Fig. 1). All components have been demonstrated in cell phones or in the laboratory. Development would be required to adapt these technologies to the space environment.

We use MEMS technology to manipulate a small sail to control roll, pitch, and yaw enables a simple design and an agile spacecraft. With lightweight electronics, sail diameters between one and five meters allow for sails from many spacecraft to be stacked on top of each other with no need for folding and associated deployment hardware. 
Initial designs with off-the-shelf components and sail diameters between one and two meters will have a mass loading of roughly 10 grams $/ \mathrm{m} 2$ and characteristic acceleration of $1 \mathrm{~mm} / \mathrm{s}^{2}$, enough be able to reach some comets and near earth objects within a few years. Optimized designs, with custom lightweight electronics and power systems and five meter

sail diameters will have characteristic accelerations close to $6 \mathrm{~mm} / \mathrm{s}^{2}$ and be able to reach most objects with semi-major axis below four AU within a few years.

\section{Mission profile}

Mission durations would be in the range of 2-8 years. For example, we have estimated a trajectory for a sample return mission to JFC 67P/Churyumov-Gerasimenko with a 5mdiameter solar sail launched in 2025 would require two years from launch to recovery.

\subsection{Launch and deployment}

A ride-share spacecraft (a "hive") could carry thousands of BLISS spacecraft to GEO, thus bypassing the hazardous Van Allen belts and the slow ascent from LEO to GEO using solar propulsion.

\subsection{De-tumble and Orient}

After release from the hive, the BLISS spacecraft will need to kill any residual rotation imparted by release, or by the spring energy that unfolds the sail. Orientation control of the spacecraft is provided by MEMS inchworm motors pulling on shroud lines on the sail, as described below. For rotation rates above one degree per second, the on-board IMU will inform the control system's decisions. Low-cost MEMS gyros have thermal-noise-limited resolution and bias drift of a small fraction of a degree per second. Below that rate, the camera will be used in a time lapse exposure to find the axis of rotation and rotation rate. Once the rotation rate is sufficiently low, a standard "lost in space" algorithm will be run on the star images to accurately determine orientation. If there is sufficiently low magnitude and duration of tumbling on release, the Inertial Measurement Unit (IMU) itself will provide sufficiently accurate initial orientation estimates. During this initial phase, communication between spacecraft and with the hive will be possible to a distance of a few hundred meters using the integrated WiFi radio available in almost all embedded LINUX platforms.

\subsection{Near-Earth Communication}

Within a range of $\sim 1$ million $\mathrm{km}$, communication between spacecraft, and communication between individual spacecraft and earth, will be via steered semiconductor laser transmitters and SPAD receivers. 


\subsection{Spiral out to interplanetary space}

Once oriented, the spacecraft will begin the process of increasing its orbital altitude until it crosses out of the earth's sphere of influence. The approximate escape time is given by [5]

$$
t_{\text {escape }}=\frac{2800 \text { days }}{\beta \sqrt{6371+h(\mathrm{~km})}}
$$

where $h$ is the initial orbital altitude, and $\beta$ is the sail lightness number, the ratio of the maximum light pressure acceleration to the solar gravitation, which is independent of distance from the sun. For a sail mass loading of $10 \mathrm{~g} / \mathrm{m}^{2}, \beta \approx 0.16$. Starting from GEO this process takes about three months.

Imaging the earth, moon, and stars provides the necessary position input for the attitude control system to calculate the necessary sail angle during this process. The optimal sail normal relative to the sun (called the cone angle) varies from $0^{\circ}$ to $90^{\circ}$ and then $-90^{\circ}$ to $0^{\circ}$ throughout the course of each orbit. The transition from $+90^{\circ}$ to $-90^{\circ}$ degrees needs to happen very quickly relative to the 24 hour orbit. Peak angular acceleration scales as roughly the reciprocal of sail diameter. For a 5 meter diameter sail, the time to perform a flip is roughly an hour. For a 1 meter diameter sail it is roughly ten minutes.

\subsection{Interplanetary navigation}

Beyond a range of $\sim 1$ million $\mathrm{km}$, the spacecraft will be out of communication with ground stations, so will be autonomous. Interplanetary navigation will rely on imaging of inner planets against the stellar background. Location accuracy using this method is expected to be better than $0.01 \mathrm{AU}$.

\subsection{Near-comet Mission Ops}

Once it is close to the target comet nucleus, the spacecraft will use its camera to do terminal guidance. At $0.01 \mathrm{AU}$ distance from the spacecraft, a $3 \mathrm{~km}$ diameter comet $1 \mathrm{AU}$ from the sun will have an apparent magnitude of 5 to 7 . So the spacecraft will get close enough to the comet by navigating based on sightings of the planets for visual acquisition of the comet with the camera.

\subsection{Return and Recovery}

The hive would be used to collect returning BLISS spacecraft. The hive will remain in a fixed orbit in or near GEO, and the BLISS spacecraft would navigate to the hive for collection. Because of the $240 \mathrm{msec}$ round-trip latency for GEO, the BLISS spacecraft collection could be done by a ground-based human operator using an arm on the hive equipped with electromagnets. Sails could be jettisoned or removed before stowage of the spacecraft in the hive. The hive will return the BLISS spacecraft to Earth in a Stardust-like sample return capsule, or, if available, it could be recovered by a next-generation vehicle such as the SpaceX Starship. 


\section{Master Equipment List (MEL)}

\subsection{Digital processing and storage}

Cell phones and the internet of things have led to a wide range of choices in energyefficient, physically small computation platforms. The candidate platform should have the computational capabilities to store and process high resolution images. Other storage and processing requirements, such as guidance navigation and control, and communication processing, place significantly lower demands on processing and storage. Even the image processing task has relatively low requirements, with allowable processing times on the order of minutes or perhaps even hours.

Significant overkill for these requirements is available in a variety of single-board computers, of which the Gumstix Overo Water is a representative. This $\$ 150$ computer runs Linux on a $720 \mathrm{MHz}$ ARM Cortex A8 with 1 GB of flash memory and $512 \mathrm{MB}$ of DRAM. The entire computer weighs 5.6 grams, including connectors for camera and power, empty board space, and a microSD card slot. Adding a $512 \mathrm{~GB}$ card to store images and other data increases the system weight by roughly 0.25 grams. The processor burns roughly $1 \mathrm{~W}$ peak, and has many power control modes. It is built from components rated from 0 to $85 \mathrm{C}$.

Similar computational capability in a carefully designed board with off-the-shelf chips could weigh roughly a gram, and operate from $-40 \mathrm{C}$ to $+85 \mathrm{C}$. Custom designed silicon would drop the mass to well under a gram, and increase the allowed temperature range.

\subsection{Attitude control}

A microelectromechanical systems (MEMS) Inertial Measurement Unit (IMU) will provide high-rate rotation sensing, and the CMOS camera will provide low-rate rotation sensing, along with star tracking and attitude determination, and rough localization.

\subsection{Navigation}

Spacecraft will rely on triangulation using the location of the planets against the stellar background. Unmodified cell phone cameras are able to take pictures of stars down to visual magnitude 5 through earth's atmosphere. With minimal effort the inner six planets can be located in these images with an accuracy of roughly one milliradian. This will allow a spacecraft to localize itself in interplanetary space to better than $0.01 \mathrm{AU}$ at a single point in time using several photographs. With better image processing algorithms and fusion of sensor data taken over many days, it is likely that this accuracy will improve by at least one order of magnitude.

\subsection{Propulsion}

Propulsion will be provided by a solar sail, with MEMS motors controlling the sail and spacecraft orientation in 3 axes. Orientation control of the spacecraft is provided by MEMS inchworm motors pulling on shroud lines on the sail. For a cometary sample return mission, a 1-5 m diameter solar sail is required. Aluminized mylar is already available in $1.5 \mu \mathrm{m}$ 
thickness and yet is strong enough to be handled, cut, folded, and thermally bonded without the need for extraordinary care. An aluminized mylar sail with a bent carbon fiber ring on the perimeter is sufficiently flexible to be bent into a multi-turn flattened disk, and sufficiently stiff to pop back to regular shape after release on orbit. Automobile dashboard shades and small camping tents are often folded in a similar manner. For a launch with one thousand spacecraft after the technology has been proven, the sails may be stored stacked on top of each other, with the spacecraft bodies arrayed around the perimeter. Note that one thousand spacecraft of this type will weigh roughly $10 \mathrm{~kg}$.

The sail will be connected to the spacecraft body with three carbon fiber rods. One of these rods will be of fixed length, and the other two will be attached to MEMS linear inchworm motors on the spacecraft body [6]. Other lightweight motors are possible. Parallel and differential actuation of these linear motors will move the sail normal relative to the center of mass of the system. Motion of the sail normal will provide a moment to the center of mass, allowing control of pitch and yaw. A fourth rod, with the third motor, will control a separate section of the sail to generate a moment about the roll axis.

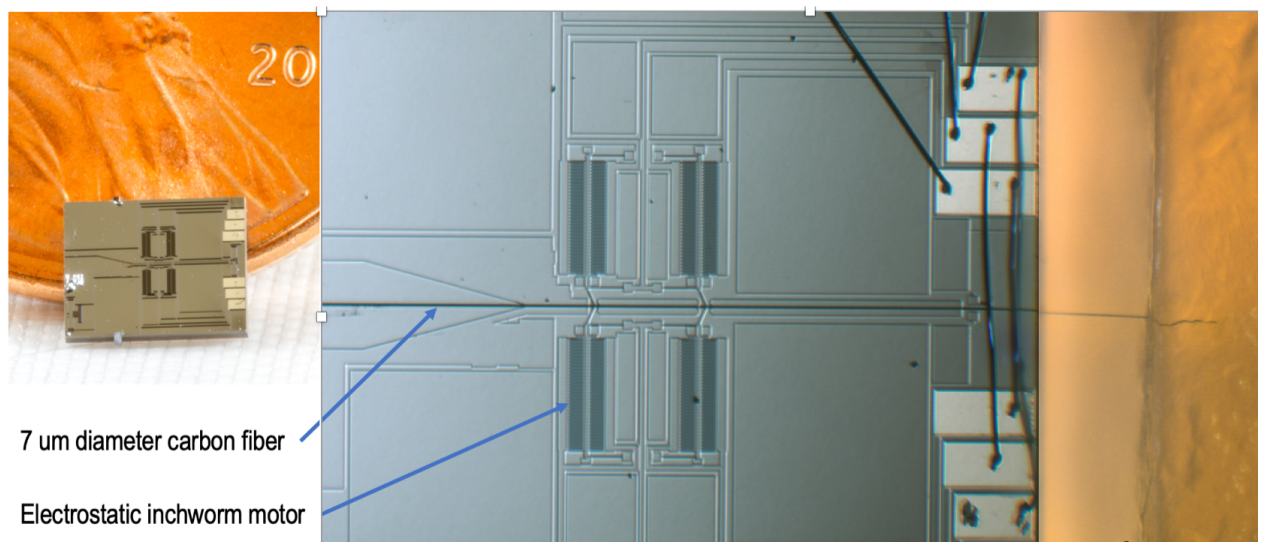

Figure 2: A small electrostatic inchworm motor driving a carbon fiber into agar. [6]

\subsection{Communication}

A standard wi-fi radio with a range of several hundred $m$ will be used for near-hive communications. A commercial $5 \mathrm{~W}$ edge emitting laser with a $1 \mathrm{~cm}$ aperture and a SPAD with a $10 \mathrm{~cm}^{2}$ Fresnel lens and $35 \%$ photon detection probability will achieve a bit rate in excess of $100 \mathrm{kbps}$ at a distance of one million kilometers using pulse position modulation and an average power dissipation of under $2 \mathrm{~W}$ at the transmitter. Prototypes of systems with similar size scale and pointing accuracy have previously been demonstrated [7, 8].

\subsection{Power}

Power will be provided by a $10 \mathrm{~cm} \times 10 \mathrm{~cm}$ primary solar panel, a small rechargeable lithium battery, and small solar cells distributed around the structure to maintain power during maneuvers. 


\subsection{Thermal control}

Thermal control will be accomplished by control of the orientation relative to the sun of the primary solar cells, a small solar reflector, and an HOPG radiator fin, in addition to management of the electronics power consumption.

\section{If NASA doesn't do it, someone else will}

Almost all of the components required to build and deploy swarms of miniature interplanetary spacecraft already exist the pockets of almost everyone on the planet. The few additional components that are required, for example the MEMS inchworm motors, are unlikely to be problematic to implement in a spacecraft application. As a result, the barrier to entry is low, and it is highly likely that swarms of miniature interplanetary spacecraft will be deployed by one or more commercial or state player within the next decade. A high-level question for NASA to consider is what this may mean for its planetary and heliospheric programs, and whether investment in space qualification of the critical components is warranted.

\section{References}

[1] Óro et al. Mechanism of synthesis of adenine from hydrogen cyanide under possible primitive earth conditions. Nature, 190(1):389, 1961.

[2] C. Chyba, C. \& Sagan. Endogenous production, exogenous delivery and impact-shock synthesis of organic molecules: an inventory for the origins of life. Nature, 355(1):125, 1992.

[3] C. Engrand S. A. Sandford and A. Rotundi. 'Elements, 12(1):185, 2016.

[4] R. C. et al. Ogliore. Incorporation of a late-forming chondrule into comet wild 2. Astrophysical Journal Lett., 745L(1):19, 2012.

[5] Colin R McInnes. Solar sailing: technology, dynamics and mission applications. Springer Science \& Business Media, 2013.

[6] Rachel S Zoll, Craig B Schindler, Travis L Massey, Daniel S Drew, Michel M Maharbiz, and Kristofer SJ Pister. Mems-actuated carbon fiber microelectrode for neural recording. IEEE transactions on nanobioscience, 18(2):234-239, 2019.

[7] Matt Last, Brian S Leibowitz, Baris Cagdaser, Anand Jog, Lixia Zhou, B Boser, and Kristofer SJ Pister. Toward a wireless optical communication link between two small unmanned aerial vehicles. In Proceedings of the 2003 International Symposium on Circuits and Systems, 2003. ISCAS'03., volume 3, pages III-III. IEEE, 2003.

[8] Brian S Leibowitz, Bernhard E Boser, and Kristofer SJ Pister. A 256-element cmos imaging receiver for free-space optical communication. IEEE journal of solid-state circuits, 40(9):1948-1956, 2005. 